\title{
EFFECT OF ANTI-BIOFILM POTENTIAL OF DIFFERENT MEDICINAL PLANTS: REVIEW
}

\author{
ABINAYA MANI, GAYATHRI MAHALINGAM* \\ Department of Biotechnology, School of Bioscience and Technology, VIT University, Vellore - 632 014, Tamil Nadu, India. \\ Email: gayathrigopinath@vit.ac.in
}

Received: 23 September 2016, Revised and Accepted: 29 October 2016

ABSTRACT

Medicinal plant products are the natural products which have been very useful for human to cure various ailments and as an alternative medicine for conventional therapy. However, bacteria in natural environments are mainly exist in biofilm formation and are more susceptible to cause severe infections than the planktonic counterparts. Biofilm is associated with impaired epithelization and granulation tissue formation and also promotes a low-grade inflammatory response that interferes with wound healing. Since the infection caused by biofilm is often very difficult to treat, there is a need to find a new active anti-biofilm agent. In recent past, interest in the therapeutic and nutritional properties of various medicinal plants and its natural phytochemical compounds which have established for their anti-biofilm activities has been increased gradually. In this review, we have described various aerial parts of medicinal plants which have anti-biofilm effect which was evaluated against biofilm producing different bacterial pathogens and antimicrobial agents which are responsible to cure wound healing

Keywords: Medicinal plants, Phytochemical, Anti-biofilm activity.

(C) 2017 The Authors. Published by Innovare Academic Sciences Pvt Ltd. This is an open access article under the CC BY license (http://creativecommons. org/licenses/by/4. 0/) DOI: http://dx.doi.org/10.22159/ajpcr.2017.v10i2.15334

\section{INTRODUCTION}

Bacterial biofilm is defined as microbes derived sessile communities of microorganism attached to the living or inert surface and is embedded in the extracellular polymeric substances (EPS) [1]. The role of the biofilm is to attach to the epithelia layer of multicellular organisms, abiotic surfaces, and interfaces between air and water. Some bacterial biofilms have been reported to have useful effects on food chains, sewage treatment of plants, to eliminate petroleum oil or hydrocarbon spillage from the oceans and human chronic bacterial infections. In 1970s, the research on biofilm has been extensive, with significant evidence showing that bacteria exist predominantly as a biofilm phenotype in medical, natural and industrial ecosystem [2]. Today, biofilm is implicated in numerous bacterial infections including those associated with the urinary tract, ear, sinuses, cystic fibrosis, indwelling catheters, chronic wounds, and periodontal disease.

Biofilm is often characterized by surface attachment, structural heterogenicity, genetic diversity, complex community interaction, and an extracellular matrix of polymeric substances. Single celled organisms generally exhibit two different modes of behaviors [3]. Mostly bacterial embedded biofilms arrive in a stationary phase, during these phase physicochemical interactions take place and produced a slime layer, followed by these lower diffusion of active molecules, and then a lesser susceptibility of antimicrobial killing [4]. The bacterial biofilm shows a different metabolic state than planktonic bacteria, mainly during transcription and cell interaction. The mode of biofilm growth results in an increased bacterial resistance against antimicrobial agent and host defense mechanism is now well-documented, and these was highlights the importance of effective biofilm management in chronic infections [3].

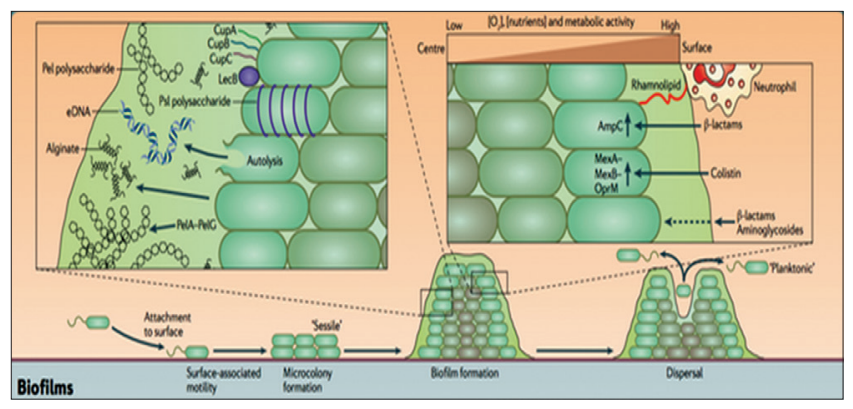

\section{LIFE CYCLE OF BIOFILMS}

The life cycle of biofilm contains four general stages: First the cells are adhere to a substrate such as polysaccharides or glycoprotein and form microcolonies [5]. As more as cell aggregates, the concentration of chemical signals reaches a point that triggers genetic changes in the cells that cause them to bind tightly to the surface and neighboring cells. These microcolonies produce a thick extracellular matrix composed of exopolysaccharides (EPS), protein, extracellular DNA and other polymer that forms a protective physical barrier around the bacteria, allowing them to grow into a mature biofilm of complex communities that are capable of chemical communication, a process called quorum sensing (QS). QS molecules have been shown to be essential for biofilm formation $[6,7]$.

Once the biofilm reaches particular cell density, point of saturation, biofilm turns off the expression of gene producing EPS products and reactivates flagella motility genes to disperse new planktonic cells from disseminating biofilm in search of new environment [8]. The relationship between the host and its microbial communities is carefully balanced, but under certain conditions, it can break down and result in infectious diseases. Microbes of biofilm secrete specific toxins, generate a hypoxemic microenvironment, and the host immune system, from all of which may contribute to delayed wound healing [9]. According to a recent publication announcement from the National Institutes of Health, more than $60 \%$ of all microbial infections are caused by biofilms [10].

The exploration for alternative therapies is a required and using nowadays, for examples, animal or natural plant products, and/or combination with antibiotics or synthetic compounds seems to be one of the auspicious solutions [11]. Drugs which are derived from natural sources are major interest, and thus they are focused for activity against biofilm producing microorganism. Till now, $80 \%$ of human bacterial infections are formed by biofilm-associated, mostly infections are frequently being caused by Staphylococcus epidermidis, Staphylococcus aureus, Escherichia coli, and Pseudomonas aeruginosa [12].

Plant-based compounds are widely accepted due to the perception that they are safe, easily available, less expensive, and also have no side effects. Plant-based drugs have greater potential for producing new 
drugs and used in traditional medicine to treat the immune booster, chronic and several infectious diseases. The World Health Organization estimated that about $80 \%$ of the world populations depends on traditional herbal medicine for their primary healthcare [13]. There are approximately 500,000 plant species occurring worldwide and $<1 \%$ has been screened for biological active compound [14].

Plant has the ability to synthesize a wide variety of phytochemical compounds as secondary metabolites. Many of the phytochemical have been used to effectively treatment of various ailments for humanity. The different chemical and components extracts of many plants have been proved to be possible sources for new drugs [5]. The plantderived substances are under research for possible application in pharmaceutical industry includes crude extracts of leaves, stems, barks, root, essential oils, and novel compounds isolated from any of these sources. The effects of plant extracts to prevent biofilm formation and adherence have been shown in earlier studies [8].

Nowadays biofilm is considered major target for pharmacological development of drugs. For example, in future it may be possible to formulate wound care products, such as rinses or dressing, debridement pads, gel, anti-biofilm agent that penetrate through biofilm EPS, thus exposing the bacteria and increasing their susceptibility to antimicrobials. This review can be contribute to the development of a new approach to prevent and to treat biofilm infections (Table 1).

\section{CONCLUSION}

Large group of traditional medicinal plants is used for different medicinal properties, which have a greater potential to cure various diseases. Furthermore, various extracts from different medicinal plant parts such as leaves, flowers, essential oils, root, and barks were also found to possess the anti-biofilm activity. Nowadays, it is known that resistance to antibiotic is often caused by biofilm formation of microbial pathogens. Therefore, the development of effective and safe medicine properties of plant extracts, which have antimicrobial activity have developed in both academic and industrial sectors. In this review, we have described that various aerial parts of traditional medicinal plants were able to inhibit the biofilm formation from various strains, were isolated from different infection sources such as wound, septicemia, urinary tract infections showing its potential value as an alternative to anti-infection agent. Thus, there remaining tremendous scope for a further research study of these traditional medicinal plants to establish their therapeutic efficacy and molecular mechanism of anti-biofilm effect of the bioactive compound.

Table 1: List of medicinal plants which have anti-biofilm activity

\begin{tabular}{|c|c|c|c|c|c|c|}
\hline Name & $\begin{array}{l}\text { Parts } \\
\text { used }\end{array}$ & $\begin{array}{l}\text { Types of plant } \\
\text { extracts }\end{array}$ & $\begin{array}{l}\text { Medicinal uses of } \\
\text { plant }\end{array}$ & $\begin{array}{l}\text { Pathogens used for } \\
\text { biofilm }\end{array}$ & Activities & References \\
\hline $\begin{array}{l}\text { Azadirachta } \\
\text { indica }\end{array}$ & Leaves & Methanol & $\begin{array}{l}\text { Various ailments, } \\
\text { act as contraceptive } \\
\text { and sedative agent }\end{array}$ & $\begin{array}{l}\text { Escherichia coli, } \\
\text { Pseudomonas aeruginosa }\end{array}$ & $\begin{array}{l}\text { Antibacterial, } \\
\text { anthelmintic and } \\
\text { antibiofilm activities }\end{array}$ & {$[15,16]$} \\
\hline Vitex negundo & Leaves & $\begin{array}{l}\text { Methanol, } \\
\text { ethyl acetate, } \\
\text { petroleum ether, } \\
\text { and hexane }\end{array}$ & $\begin{array}{l}\text { Wound, ulcer, } \\
\text { asthma, ophthalmic, } \\
\text { verminosis, } \\
\text { bronchial smooth } \\
\text { muscles relaxant, } \\
\text { nervine and } \\
\text { aphrodisiac }\end{array}$ & Escherichia coli & $\begin{array}{l}\text { Antinociceptive, } \\
\text { antimicrobial, } \\
\text { anti-inflammatory, } \\
\text { antipyretic, } \\
\text { anticonvulsant, } \\
\text { insecticidal and } \\
\text { pesticidal activities }\end{array}$ & [15] \\
\hline $\begin{array}{l}\text { Tridax } \\
\text { procumbens }\end{array}$ & Leaves & $\begin{array}{l}\text { Methanol, ethyl } \\
\text { acetate }\end{array}$ & $\begin{array}{l}\text { Bronchial, } \\
\text { dysentery, hair } \\
\text { growth promotes, } \\
\text { and insect repellent }\end{array}$ & Escherichia coli & $\begin{array}{l}\text { Immunomodulatory, } \\
\text { wound healing, } \\
\text { hepatoprotective, } \\
\text { antidiabetic, antiobesity, } \\
\text { antimicrobial and } \\
\text { anti-inflammatory } \\
\text { activities }\end{array}$ & {$[15,17]$} \\
\hline $\begin{array}{l}\text { Ocimum } \\
\text { tenuiflorumi }\end{array}$ & Leaves & Methanol & $\begin{array}{l}\text { Coughs, colds, } \\
\text { vomiting, flatulence, } \\
\text { heal peptic ulcers }\end{array}$ & Escherichia coli & $\begin{array}{l}\text { Wound healing effect, } \\
\text { radio protective, } \\
\text { anti-carcinogenic } \\
\text { properties, } \\
\text { anti genotoxic, } \\
\text { neuroprotective effect, } \\
\text { and larvicidal property }\end{array}$ & [15] \\
\hline $\begin{array}{l}\text { Piper lonum } \\
\text { Piper nigrum }\end{array}$ & Grains & $\begin{array}{l}\text { Methanol, } \\
\text { petroleum ether, } \\
\text { chloroform and } \\
\text { ethyl acetate }\end{array}$ & $\begin{array}{l}\text { Malaria, asthma, } \\
\text { cough, diabetes and } \\
\text { heart problems }\end{array}$ & Streptococcus pyogens & $\begin{array}{l}\text { Anti-biofilm, } \\
\text { antibacterial, } \\
\text { antioxidant activities }\end{array}$ & [18] \\
\hline $\begin{array}{l}\text { Pittosporum } \\
\text { tetraspermum }\end{array}$ & Leaves & Ethyl acetate & $\begin{array}{l}\text { Chronic bronchitis, } \\
\text { rheumatism, skin } \\
\text { diseases, cutaneous } \\
\text { diseases and } \\
\text { leprosy }\end{array}$ & $\begin{array}{l}\text { Escherichia coli, } \\
\text { Salmonella typhi, } \\
\text { Pseudomonas } \\
\text { aueroginasa }\end{array}$ & $\begin{array}{l}\text { Antibacterial, antifungal, } \\
\text { anti-biofilm, antioxidant } \\
\text { and anticancer activities }\end{array}$ & [19] \\
\hline $\begin{array}{l}\text { Couroupita } \\
\text { guianensis }\end{array}$ & Fruits & Chloroform & $\begin{array}{l}\text { Tumors, pain and } \\
\text { hypertension }\end{array}$ & $\begin{array}{l}\text { Pseudomonas } \\
\text { aueroginasa }\end{array}$ & $\begin{array}{l}\text { Antimicrobial, } \\
\text { antimycobacterial, } \\
\text { antifungal, antiseptic, } \\
\text { antibiotic, anti-biofilm } \\
\text { anti-inflammatory } \\
\text { activities. }\end{array}$ & {$[20]$} \\
\hline
\end{tabular}


Table 1: (Continued)

\begin{tabular}{|c|c|c|c|c|c|c|}
\hline Name & $\begin{array}{l}\text { Parts } \\
\text { used }\end{array}$ & $\begin{array}{l}\text { Types of plant } \\
\text { extracts }\end{array}$ & $\begin{array}{l}\text { Medicinal uses of } \\
\text { plant }\end{array}$ & $\begin{array}{l}\text { Pathogens used for } \\
\text { biofilm }\end{array}$ & Activities & References \\
\hline $\begin{array}{l}\text { Chamaemelum } \\
\text { nobile }\end{array}$ & Flowers & Aqueous & $\begin{array}{l}\text { Malaria, peptic } \\
\text { ulcers, wound } \\
\text { healing }\end{array}$ & $\begin{array}{l}\text { Pseudomonas } \\
\text { aueroginasa }\end{array}$ & $\begin{array}{l}\text { Anti-inflammatory, } \\
\text { antimicrobial, antiseptic, } \\
\text { anti-swarming activities }\end{array}$ & [21] \\
\hline Vinca minor & Leaves & $\begin{array}{l}\text { Aquatic, acetone } \\
\text { and ethyl acetate }\end{array}$ & $\begin{array}{l}\text { Supporting brain } \\
\text { metabolism }\end{array}$ & $\begin{array}{l}\text { Proteus mirabilis, } \\
\text { Pseudomonas } \\
\text { aueroginasa }\end{array}$ & $\begin{array}{l}\text { Antimicrobial, } \\
\text { anti-biofilm activities }\end{array}$ & [22] \\
\hline $\begin{array}{l}\text { Melissa } \\
\text { officinalis }\end{array}$ & $\begin{array}{l}\text { Essential } \\
\text { oils }\end{array}$ & Liquid & $\begin{array}{l}\text { Gastrointestinal } \\
\text { disorders, stress, } \\
\text { sleep disorder, } \\
\text { spasmolytic, } \\
\text { Alzheimer diseases }\end{array}$ & $\begin{array}{l}\text { Escherichia coli, } \\
\text { Staphylococcus aureus }\end{array}$ & $\begin{array}{l}\text { Antibacterial and } \\
\text { antifungal }\end{array}$ & {$[23,24]$} \\
\hline $\begin{array}{l}\text { Ficus } \\
\text { sansibarica }\end{array}$ & $\begin{array}{l}\text { Fruits, } \\
\text { leaves, } \\
\text { stem barks }\end{array}$ & $\begin{array}{l}\text { Cold extract } \\
\text { methanol, } \\
\text { hexane, } \\
\text { dichloromethane, } \\
\text { ethyl acetate and } \\
\text { methanol }\end{array}$ & $\begin{array}{l}\text { Wound healing, } \\
\text { tuberculosis, } \\
\text { diabetes malaria } \\
\text { and HIV }\end{array}$ & $\begin{array}{l}\text { Escherichia coli, } \\
\text { Staphylococcus aureus }\end{array}$ & $\begin{array}{l}\text { Antioxidant, } \\
\text { antibacterial, antifungal, } \\
\text { antidiabetics, anticancer, } \\
\text { anti-inflammatory and } \\
\text { antihyperglycemia }\end{array}$ & [25] \\
\hline $\begin{array}{l}\text { Pongamia } \\
\text { pinnata }\end{array}$ & Leaves & Methanol & $\begin{array}{l}\text { Antihelminthitic, } \\
\text { gonorrhea, leprosy, } \\
\text { inflammation, piles } \\
\text { and wound }\end{array}$ & Streptococcus mutans & $\begin{array}{l}\text { Antiplasmodial, } \\
\text { anti-inflammatory, } \\
\text { antidiarrhoeal, } \\
\text { antioxidant, } \\
\text { anti-hyperammonemi, } \\
\text { antiulcer, } \\
\text { antihyperglycaemic and } \\
\text { antilipidperoxidative } \\
\text { activities }\end{array}$ & [26] \\
\hline $\begin{array}{l}\text { Coriandrum } \\
\text { sativum }\end{array}$ & Seed & $\begin{array}{l}\text { Ethanol and } \\
\text { water }\end{array}$ & $\begin{array}{l}\text { Drug for } \\
\text { indigestions, } \\
\text { worms, pain } \\
\text { in joints and } \\
\text { rheumatism }\end{array}$ & Staphylococcus aureus & Antibiofilm activity & [28] \\
\hline Leucas aspera & $\begin{array}{l}\text { Whole } \\
\text { plant }\end{array}$ & $\begin{array}{l}\text { Methanol, } \\
\text { ethyl acetate, } \\
\text { petroleum ether, } \\
\text { and hexane }\end{array}$ & $\begin{array}{l}\text { Scabies, psoriasis, } \\
\text { snake bite, laxative, } \\
\text { and diaphoretic }\end{array}$ & $\begin{array}{l}\text { Streptococcus } \\
\text { pyogenes, Pseudomonas } \\
\text { aeruginosa, } \\
\text { Staphylococcus aureus }\end{array}$ & $\begin{array}{l}\text { Antibacterial, } \\
\text { anti-inflammatory, } \\
\text { antidiarrheal, } \\
\text { antioxidant, } \\
\text { antibacterial, } \\
\text { hepatoprotective, } \\
\text { anti-diabetic activities } \\
\text { and central nervous } \\
\text { system activity }\end{array}$ & [29] \\
\hline $\begin{array}{l}\text { Artocarpus } \\
\text { lakoocha }\end{array}$ & Bark & Aqueous extracts & $\begin{array}{l}\text { Wound, skin lesion, } \\
\text { and cosmetic }\end{array}$ & $\begin{array}{l}\text { Candida albicans, } \\
\text { Candida tropicalis, } \\
\text { Candida dubliniensis }\end{array}$ & $\begin{array}{l}\text { Antiviral, antibiofilm, } \\
\text { anticandidal, } \\
\text { anti-inflammatory, } \\
\text { anticancer activities, } \\
\text { anti-HIV properties, } \\
\text { anti-skin aging and } \\
\text { atherosclerosis }\end{array}$ & {$[30,31]$} \\
\hline $\begin{array}{l}\text { Murraya } \\
\text { koenigii }\end{array}$ & $\begin{array}{l}\text { Leaves, } \\
\text { essential } \\
\text { oil }\end{array}$ & Ethanol, aqueous & $\begin{array}{l}\text { Tonic, chronic } \\
\text { wound, stomachic, } \\
\text { inflammation, } \\
\text { itching, vomiting, } \\
\text { cure piles }\end{array}$ & Pseudomonas aeruginosa & $\begin{array}{l}\text { Anti-biofilm, antiulcer, } \\
\text { antidiarrhoea, } \\
\text { antidiabetics activities, } \\
\text { cholesterol reducing } \\
\text { property, phagocytic } \\
\text { activity, and more useful } \\
\text { medicinal properties }\end{array}$ & {$[32,33]$} \\
\hline
\end{tabular}


Table 1: (Continued)

\begin{tabular}{|c|c|c|c|c|c|c|}
\hline Name & $\begin{array}{l}\text { Parts } \\
\text { used }\end{array}$ & $\begin{array}{l}\text { Types of plant } \\
\text { extracts }\end{array}$ & $\begin{array}{l}\text { Medicinal uses of } \\
\text { plant }\end{array}$ & $\begin{array}{l}\text { Pathogens used for } \\
\text { biofilm }\end{array}$ & Activities & References \\
\hline Hakea sericea & $\begin{array}{l}\text { Aerial } \\
\text { parts }\end{array}$ & Methanol & No medicinal uses & $\begin{array}{l}\text { Staphylococcus aureus } \\
\text { MRSA }\end{array}$ & $\begin{array}{l}\text { Antioxidant, } \\
\text { antibacterial, } \\
\text { anti-biofilm and } \\
\text { cytotoxicity activities }\end{array}$ & {$[34]$} \\
\hline $\begin{array}{l}\text { Chromolaena } \\
\text { odorata }\end{array}$ & Leave & $\begin{array}{l}\text { Chloroform, } \\
\text { ethanol }\end{array}$ & Cough, malaria & Pseudomonas aeruginosa & $\begin{array}{l}\text { Antibacterial, } \\
\text { antioxidant, anti-biofilm, } \\
\text { anti-inflammatory, } \\
\text { wound healing }\end{array}$ & [35] \\
\hline $\begin{array}{l}\text { Terminalia } \\
\text { fagifolia }\end{array}$ & Stem bark & Ethanol & $\begin{array}{l}\text { Gastrointestinal } \\
\text { disturbances, such } \\
\text { as ulcer, gastritis, } \\
\text { and diarrhea }\end{array}$ & $\begin{array}{l}\text { Staphylococcus aureus, } \\
\text { Staphylococcus } \\
\text { epidermidis }\end{array}$ & $\begin{array}{l}\text { Antibacterial, } \\
\text { antibiofilm, antioxidant, } \\
\text { gastric antiulcerogenic, } \\
\text { and hypokinetics } \\
\text { activities. In vitro and } \\
\text { in vivo cytotoxicity } \\
\text { studies }\end{array}$ & [36] \\
\hline $\begin{array}{l}\text { Pityrocarpamo } \\
\text { moniliformis }\end{array}$ & Leaves & Aqueous & Healing process & $\begin{array}{l}\text { Staphylococcus } \\
\text { epidermidis, } \\
\text { Pseudomonas aeruginosa }\end{array}$ & $\begin{array}{l}\text { Anti-biofilm, } \\
\text { antibacterial activities, } \\
\text { antinociceptive, } \\
\text { anti-inflammatory } \\
\text { agent }\end{array}$ & {$[37,38]$} \\
\hline $\begin{array}{l}\text { Myracrodruoun } \\
\text { urundeuva }\end{array}$ & $\begin{array}{l}\text { Leaves, } \\
\text { branches, } \\
\text { and steam } \\
\text { bark }\end{array}$ & Aqueous & $\begin{array}{l}\text { Tumors, } \\
\text { rheumatism, } \\
\text { inflammations, } \\
\text { acne, pain, skin } \\
\text { problems, allergy, } \\
\text { cracks, etc., }\end{array}$ & $\begin{array}{l}\text { Staphylococcus } \\
\text { epidermidis }\end{array}$ & $\begin{array}{l}\text { Anti-biofilm, } \\
\text { anti-inflammatory, } \\
\text { anti-allergic, and wound } \\
\text { healing properties }\end{array}$ & [39] \\
\hline $\begin{array}{l}\text { Agrimonia } \\
\text { eupatoria }\end{array}$ & Flowers & $\begin{array}{l}\text { Ethanol, diethyl } \\
\text { ether, water, and } \\
\text { acetone }\end{array}$ & $\begin{array}{l}\text { Urinary tract } \\
\text { disorders }\end{array}$ & $\begin{array}{l}\text { Pseudomonas } \\
\text { aeruginosa, Proteus } \\
\text { mirabilis }\end{array}$ & $\begin{array}{l}\text { Anti-inflammatory, } \\
\text { neuroprotective, } \\
\text { antidiabetics, } \\
\text { anticancer, antiobesity } \\
\text { properties, antibiofilm, } \\
\text { antibacterial, } \\
\text { antioxidant activities }\end{array}$ & {$[40]$} \\
\hline Ibicella lutea & Aerial part & Chloroform & Skin infection & Proteus mirabilis & $\begin{array}{l}\text { Biofilm formation, } \\
\text { hemagglutination, } \\
\text { hemolysis, antibacterial } \\
\text { activity }\end{array}$ & {$[41]$} \\
\hline $\begin{array}{l}\text { Myroxylon } \\
\text { peruiferum }\end{array}$ & $\begin{array}{l}\text { Leaves, } \\
\text { bark-trunk }\end{array}$ & $\begin{array}{l}\text { Hydroalcoholic } \\
\text { extracts }\end{array}$ & $\begin{array}{l}\text { Heal new } \\
\text { wound, asthma, } \\
\text { cold, diarrhea, } \\
\text { skin parasites, } \\
\text { rheumatism and } \\
\text { urinary infection }\end{array}$ & $\begin{array}{l}\text { Staphylococcus } \\
\text { epidermidis }\end{array}$ & $\begin{array}{l}\text { Anti-biofilm, } \\
\text { antibacterial activities }\end{array}$ & [42] \\
\hline $\begin{array}{l}\text { Gymnema } \\
\text { sylvestre }\end{array}$ & Leaves & Methanol & $\begin{array}{l}\text { Ailments } \\
\text { constipation, liver } \\
\text { disease, control } \\
\text { blood sugar, } \\
\text { lipid - lowering } \\
\text { agent, ailments } \\
\text { constipation, liver } \\
\text { disease, control } \\
\text { blood sugar, } \\
\text { lipid - lowering } \\
\text { agent and weight } \\
\text { loss }\end{array}$ & Streptococcus pyogenes & $\begin{array}{l}\text { Anti-biofilm, } \\
\text { antibacterial activities }\end{array}$ & [29] \\
\hline Aerva lanata & Leaves & $\begin{array}{l}\text { Methanol, } \\
\text { petroleum ether }\end{array}$ & $\begin{array}{l}\text { Headache, uterine } \\
\text { tonic, cure kidney } \\
\text { stones, jaundice, } \\
\text { dyspepsia, } \\
\text { pneumonia, typhoid, } \\
\text { and skin diseases }\end{array}$ & $\begin{array}{l}\text { Staphylococcus aureus, } \\
\text { Escherichia coli, Bacillus } \\
\text { subtilis, Proteus vulgaris }\end{array}$ & $\begin{array}{l}\text { Anti-diarrhoeal, } \\
\text { anti-hyperglycemic, } \\
\text { antioxidant, } \\
\text { anti-helmentic, } \\
\text { anti-inflammatory, } \\
\text { diuretic, anti-urolithiatic, } \\
\text { analgesic, anti-biofilm } \\
\text { activities. }\end{array}$ & {$[43,44]$} \\
\hline
\end{tabular}


Table 1: (Continued)

\begin{tabular}{|c|c|c|c|c|c|c|}
\hline Name & $\begin{array}{l}\text { Parts } \\
\text { used }\end{array}$ & $\begin{array}{l}\text { Types of plant } \\
\text { extracts }\end{array}$ & $\begin{array}{l}\text { Medicinal uses of } \\
\text { plant }\end{array}$ & $\begin{array}{l}\text { Pathogens used for } \\
\text { biofilm }\end{array}$ & Activities & References \\
\hline $\begin{array}{l}\text { Anadenanthera } \\
\text { colubrina }\end{array}$ & $\begin{array}{l}\text { Stem bark, } \\
\text { branches, } \\
\text { leaves, } \\
\text { fruits }\end{array}$ & Aqueous & $\begin{array}{l}\text { Wounds, } \\
\text { inflammation, } \\
\text { throat, lung and } \\
\text { kidney problems, } \\
\text { chest inflammation, } \\
\text { allergy, tuberculosis }\end{array}$ & $\begin{array}{l}\text { S. epidermidis, } \\
\text { Pseudomonas aeruginosa }\end{array}$ & $\begin{array}{l}\text { Anti-inflammatory, } \\
\text { antimicrobial, } \\
\text { antiproliferative } \\
\text { potential }\end{array}$ & [39] \\
\hline $\begin{array}{l}\text { Helichrysum } \\
\text { italicum }\end{array}$ & Leaf & Methanol & $\begin{array}{l}\text { Allergies, colds, } \\
\text { skin, liver, } \\
\text { gallbladder disorder, } \\
\text { inflammation, } \\
\text { sleeplessness }\end{array}$ & Pseudomonas aeruginosa & $\begin{array}{l}\text { Anti-inflammatory, } \\
\text { anti-infection properties, } \\
\text { photoprotective } \\
\text { anti-erthematous } \\
\text { activities }\end{array}$ & {$[48,49]$} \\
\hline $\begin{array}{l}\text { Vaccinium } \\
\text { vitis-idaea }\end{array}$ & Leaf & Aqueous & $\begin{array}{l}\text { Blood clots, cold, } \\
\text { kidney cyst, obesity, } \\
\text { skin diseases, } \\
\text { wounds, mycosis, } \\
\text { and anorexia }\end{array}$ & Escherichia coli & $\begin{array}{l}\text { Diuretic, diastolic, } \\
\text { diaphoretic, } \\
\text { anti-inflammatory } \\
\text { effect, antibacterial, } \\
\text { antioxidant, anticancer, } \\
\text { antiaging activities }\end{array}$ & {$[50]$} \\
\hline Euphorbia hirta & Aerial & Methanol & $\begin{array}{l}\text { Gastrointestinal, } \\
\text { bronchial, } \\
\text { parasitotosis, } \\
\text { amoebic dysentery, } \\
\text { and respiratory } \\
\text { ailments }\end{array}$ & Pseudomonas aeruginosa & $\begin{array}{l}\text { Antibacterial, } \\
\text { anti-biofilm, antioxidant, } \\
\text { antihypertensive, } \\
\text { anxiolytic, antimalarial, } \\
\text { anti-inflammatory, } \\
\text { anticancer activities. }\end{array}$ & {$[51]$} \\
\hline Arctium lappa & Leaf & Ethanol & $\begin{array}{l}\text { Diuretic, } \\
\text { diaphoretic, blood } \\
\text { purifying agent }\end{array}$ & Staphylococcus aureus & $\begin{array}{l}\text { Antibacterial, } \\
\text { antioxidant, anti-biofilm, } \\
\text { anti-inflammatory } \\
\text { activities }\end{array}$ & {$[52,53]$} \\
\hline $\begin{array}{l}\text { Aquilaria } \\
\text { crassna }\end{array}$ & Leaf & Aqueous & $\begin{array}{l}\text { Diarrhoea, } \\
\text { dysentery, skin } \\
\text { diseases, and } \\
\text { cardiovascular } \\
\text { function enhancer }\end{array}$ & $\begin{array}{l}\text { Staphylococcus } \\
\text { epidermidis }\end{array}$ & $\begin{array}{l}\text { Antibacterial, } \\
\text { antioxidant, anti-biofilm, } \\
\text { antiplasmodic, } \\
\text { antidiarrheal activities }\end{array}$ & {$[54]$} \\
\hline $\begin{array}{l}\text { Cymbopogon } \\
\text { flexuosus }\end{array}$ & $\begin{array}{l}\text { Essential } \\
\text { oil }\end{array}$ & - & $\begin{array}{l}\text { Cosmetics, } \\
\text { insecticides, } \\
\text { digestive disorder, } \\
\text { fever, and antiseptic }\end{array}$ & Staphylococcus aureus & $\begin{array}{l}\text { Antioxidant, } \\
\text { anti-inflammatory } \\
\text { activities }\end{array}$ & {$[55]$} \\
\hline Allium sativum & Leaf & $\begin{array}{l}\text { Ethanol, } \\
\text { methanol }\end{array}$ & $\begin{array}{l}\text { Wound infection, } \\
\text { common cold, } \\
\text { malaria, lung } \\
\text { tuberculosis, } \\
\text { hypertension, sexual } \\
\text { transmitted, mental } \\
\text { disorder, liver } \\
\text { diseases, asthma, } \\
\text { and diabetes }\end{array}$ & $\begin{array}{l}\text { Escherichia coli, } \\
\text { Salmonella typhi, } \\
\text { Staphylococcus aureus } \\
\text { and Bacillus cereus }\end{array}$ & $\begin{array}{l}\text { Antibacterial, } \\
\text { anti-biofilm, antifungal, } \\
\text { anti-inflammatory, } \\
\text { larvicidal activities }\end{array}$ & {$[56]$} \\
\hline
\end{tabular}


Table 1: (Continued)

\begin{tabular}{|c|c|c|c|c|c|c|}
\hline Name & $\begin{array}{l}\text { Parts } \\
\text { used }\end{array}$ & $\begin{array}{l}\text { Types of plant } \\
\text { extracts }\end{array}$ & $\begin{array}{l}\text { Medicinal uses of } \\
\text { plant }\end{array}$ & $\begin{array}{l}\text { Pathogens used for } \\
\text { biofilm }\end{array}$ & Activities & References \\
\hline Saliva triloba & $\begin{array}{l}\text { Leaves, } \\
\text { volatile oil }\end{array}$ & Ethanol & $\begin{array}{l}\text { Headaches, } \\
\text { toothaches, } \\
\text { common cold, } \\
\text { digestive problems, } \\
\text { oral infection and } \\
\text { wound healing }\end{array}$ & $\begin{array}{l}\text { Staphylococcus aureus, } \\
\text { Escherichia coli and } \\
\text { Candida albicans }\end{array}$ & $\begin{array}{l}\text { Anti-biofilm, } \\
\text { antiadhesive, anti-MRSA } \\
\text { antibacterial activities }\end{array}$ & [57] \\
\hline $\begin{array}{l}\text { Andrographis } \\
\text { paniculata }\end{array}$ & Leaves & $\begin{array}{l}\text { Ethanol, } \\
\text { methanol, } \\
\text { chloroform, } \\
\text { aqueous, and } \\
\text { hexane }\end{array}$ & $\begin{array}{l}\text { Fever, dysentery, } \\
\text { snakebite, sore } \\
\text { throat }\end{array}$ & Pseudomonas aeruginosa & $\begin{array}{l}\text { Antimicrobial, } \\
\text { antimalaria, anti-HIV } \\
\text { activities }\end{array}$ & [58] \\
\hline $\begin{array}{l}\text { Hibiscus } \\
\text { sabdariffa } \mathrm{L} .\end{array}$ & Leaves & Ethanol & $\begin{array}{l}\text { Diuretic, mild } \\
\text { laxative, cardiac, } \\
\text { nerve diseases }\end{array}$ & $\begin{array}{l}\text { Escherichia coli, } \\
\text { Pseudomonas } \\
\text { aeruginosa, } \\
\text { Staphylococcus aureus, } \\
\text { Staphylococcus } \\
\text { epidermidis, Klebsiella } \\
\text { pneumonia, Proteus } \\
\text { mirabilis }\end{array}$ & $\begin{array}{l}\text { Antibacterial, } \\
\text { anti-biofilm, antioxidant, } \\
\text { antihypertensive, } \\
\text { anticancerous activities } \\
\text { and cytotoxicity } \\
\text { properties }\end{array}$ & {$[59,60]$} \\
\hline $\begin{array}{l}\text { Mentha } \\
\text { arvensis }\end{array}$ & $\begin{array}{l}\text { Essential } \\
\text { oil from } \\
\text { leaf }\end{array}$ & - & $\begin{array}{l}\text { Indigestion, peptic } \\
\text { ulcer, skin diseases }\end{array}$ & $\begin{array}{l}\text { Aggregatibacter } \\
\text { actinomycetemcomitans }\end{array}$ & $\begin{array}{l}\text { Antibacterial, } \\
\text { anti-biofilm activities }\end{array}$ & {$[61]$} \\
\hline Mentha piperita & $\begin{array}{l}\text { Essential } \\
\text { oil from } \\
\text { leaf }\end{array}$ & - & $\begin{array}{l}\text { Toothpaste } \\
\text { digestive tablets, } \\
\text { mouthwashes }\end{array}$ & $\begin{array}{l}\text { Aggregatibacter } \\
\text { actinomycetemcomitans, } \\
\text { Candida albicans, } \\
\text { Candida dubliniensis }\end{array}$ & $\begin{array}{l}\text { Antibacterial, antifungal, } \\
\text { anti-biofilm, antiviral, } \\
\text { antifungal, antioxidant, } \\
\text { radioprotective } \\
\text { analgesic } \\
\text { activities }\end{array}$ & {$[61,62]$} \\
\hline $\begin{array}{l}\text { Lagenaria } \\
\text { sicerarita }\end{array}$ & Fruit & $\begin{array}{l}\text { Organic and } \\
\text { aqueous }\end{array}$ & $\begin{array}{l}\text { Immunosuppresent, } \\
\text { cardio-tonic, } \\
\text { cardio protective, } \\
\text { diuretic, nutritive } \\
\text { agent, purgative, } \\
\text { antidote for certain } \\
\text { poisons, emetic, } \\
\text { bronchodilator, } \\
\text { antipyretic, } \\
\text { alopecia, and } \\
\text { aphrodisiac }\end{array}$ & $\begin{array}{l}\text { Pseudomonas } \\
\text { aeruginosa, } \\
\text { Escherichia coli, } \\
\text { Staphylococcus aureus, } \\
\text { Streptococcus } \\
\text { pnueumoniae, } \\
\text { Streptococcus pyogenes }\end{array}$ & $\begin{array}{l}\text { Antibacterial, } \\
\text { antioxidant, anti-biofilm } \\
\text { activities }\end{array}$ & [63] \\
\hline $\begin{array}{l}\text { Buchanania } \\
\text { lanzan }\end{array}$ & Root & Methanol & $\begin{array}{l}\text { Digestive, curing } \\
\text { blood diseases, } \\
\text { cardiotonic, } \\
\text { astringent, } \\
\text { glandular swelling, } \\
\text { cyclophosphamide } \\
\text { induce genotoxicity, } \\
\text { oxidative stress. } \\
\text { Immunostimulant } \\
\text { and astringent } \\
\text { properties }\end{array}$ & $\begin{array}{l}\text { Escherichia coli, } \\
\text { Pseudomonas aeruginosa }\end{array}$ & $\begin{array}{l}\text { Anti-inflammatory, } \\
\text { antibacterial, } \\
\text { antioxidant activities }\end{array}$ & {$[64]$} \\
\hline $\begin{array}{l}\text { Calendula } \\
\text { officinalis }\end{array}$ & Flowers & Water & $\begin{array}{l}\text { Measles, smallpox, } \\
\text { jaundice, } \\
\text { costiveness, } \\
\text { ointment for wound, } \\
\text { ulcer, frostbite, skin } \\
\text { damage, scars, and } \\
\text { blood purification }\end{array}$ & $\begin{array}{l}\text { Salmonella, Shigella } \\
\text { dysenteriae, Shigella } \\
\text { flexneri, Shigella sonnei } \\
\text { and Escherichia coli }\end{array}$ & $\begin{array}{l}\text { Antibacterial, } \\
\text { anti-biofilm, } \\
\text { antiedematous, } \\
\text { antiseptic action, } \\
\text { antioxidant, } \\
\text { antispasmodic activities }\end{array}$ & {$[65,66]$} \\
\hline Rosa canina & Leaf & Methanol & $\begin{array}{l}\text { Cosmetic, food } \\
\text { industry, infections, } \\
\text { inflammatory } \\
\text { diseases, chronic } \\
\text { pain, flu and } \\
\text { alcoholic beverages }\end{array}$ & $\begin{array}{l}\text { Pseudomonas } \\
\text { aeruginosa, Salmonella } \\
\text { typhimurium }\end{array}$ & $\begin{array}{l}\text { Antimicrobial, } \\
\text { anti-biofilm activities }\end{array}$ & [67] \\
\hline
\end{tabular}


Table 1: (Continued)

\begin{tabular}{|c|c|c|c|c|c|c|}
\hline Name & $\begin{array}{l}\text { Parts } \\
\text { used }\end{array}$ & $\begin{array}{l}\text { Types of plant } \\
\text { extracts }\end{array}$ & $\begin{array}{l}\text { Medicinal uses of } \\
\text { plant }\end{array}$ & $\begin{array}{l}\text { Pathogens used for } \\
\text { biofilm }\end{array}$ & Activities & References \\
\hline $\begin{array}{l}\text { Rhodomyrtus } \\
\text { tomentosa }\end{array}$ & Leaf & Ethanol & $\begin{array}{l}\text { Diarrhea, wound } \\
\text { healing, urinary test } \\
\text { infections }\end{array}$ & Streptococcus pyogens & $\begin{array}{l}\text { Antioxidant, } \\
\text { antibacterial, } \\
\text { antibiofilm, } \\
\text { cancer-chemopreventive } \\
\text { activities }\end{array}$ & [68] \\
\hline $\begin{array}{l}\text { Humulus } \\
\text { lupulus L }\end{array}$ & Hop cones & Ethanol & $\begin{array}{l}\text { Nervous tension, } \\
\text { headache, } \\
\text { indigestion, } \\
\text { sedative, and } \\
\text { hypnotic }\end{array}$ & Staphylococcus aureus & $\begin{array}{l}\text { Antiadherent, } \\
\text { anti-biofilm, } \\
\text { antibacterial, } \\
\text { anti-inflammatory, } \\
\text { estrogenic activities }\end{array}$ & {$[69,70]$} \\
\hline Aegle marmelos & Leaves & Ethyl acetate & $\begin{array}{l}\text { Sore throats, cold, } \\
\text { intestinal ailments, } \\
\text { fertility, chest } \\
\text { congestion, fish } \\
\text { poison, child birth, } \\
\text { and intermittent } \\
\text { fever }\end{array}$ & $\begin{array}{l}\text { Salmonella typhi, } \\
\text { Escherichia coli, } \\
\text { Pseudomonas aerugonisa }\end{array}$ & $\begin{array}{l}\text { Antidiabetic, } \\
\text { antiulcer, antimalarial, } \\
\text { anti-inflammatory, } \\
\text { antiviral, } \\
\text { antihyperlipidaemic, } \\
\text { antibacterial, } \\
\text { antioxidant, anti-biofilm, } \\
\text { antifeedant, cytotoxic } \\
\text { activities }\end{array}$ & {$[71]$} \\
\hline $\begin{array}{l}\text { Kaempferia } \\
\text { rotunda }\end{array}$ & Rhizome & Ethanol & $\begin{array}{l}\text { Heal wounds, cure } \\
\text { stomach ailments, } \\
\text { post-delivery } \\
\text { care, blood clots, } \\
\text { jaundice, swelling }\end{array}$ & $\begin{array}{l}\text { Pseudomonas } \\
\text { aeruginosa, } \\
\text { Staphylococcus aureus }\end{array}$ & $\begin{array}{l}\text { Antitumor, antiulcer, } \\
\text { anti-inflammatory, } \\
\text { antiaging activities }\end{array}$ & [72] \\
\hline
\end{tabular}

MRSA: Methicillin-resistant Staphylococcus aureus

\section{REFERENCES}

1. Donlan RM, Costerton JW. Biofilms: Survival mechanisms of clinically relevant microorganisms. Clin Microbiol Rev 2002;15(2):167-93.

2. Costerton JW, Geesey GG, Cheng KJ. How bacteria stick. Sci Am 1978;238(1):86-95.

3. Jesaitis AJ, Franklin MJ, Berglund D, Sasaki M, Lord CI, Bleazard JB, et al. Compromised host defense on Pseudomonas aeruginosa biofilms: Characterization of neutrophil and biofilm interactions. J Immunol 2003;171(8):4329-39.

4. Gualtieri M, Bastide L, Villain-Guillot $\mathrm{P}$, Michaux-Charachon S, Latouche J, Leonetti JP. In vitro activity of a new antibacterial rhodanine derivative against Staphylococcus epidermidis biofilms. J Antimicrob Chemother 2006;58(4):778-83.

5. Hauser AR. The type III secretion system of Pseudomonas aeruginosa infection by injection. Nat Rev Microbiol 2011;7(9):654-665.

6. Chen H, Fujita M, Feng Q, Clardy J, Fink GR. Tyrosol is a quorumsensing molecule in Candida albicans. Proc Natl Acad Sci U S A 2004;101(14):5048-52.

7. Hornby JM, Nickerson KW. Enhanced production of farnesol by Candida albicans treated with four azoles. Antimicrob Agents Chemother 2004;48(6):2305-7.

8. Slonczewski JL, Foster JW. Bacterial culture, growth and development. Microbiology: An Evolving Science. Ch. 4. USA: W. W. Norton \& Company; 2013. p.115-48.

9. Zhao G, Usui ML, Underwood RA, Singh PK, James GA, Stewart PS, et al. Time course study of delayed wound healing in a biofilm-challenged diabetic mouse model. Wound Repair Regen 2012;20(3):342-52.

10. Nwodo UU, Green E, Okoh AI. Bacterial exopolysaccharides: Functionality and prospects. Int J Mol Sci 2012;13(11):14002-15.

11. Dürig A, Kouskoumvekaki I, Vejborg RM, Klemm P. Chemoinformaticsassisted development of new anti-biofilm compounds. Appl Microbiol Biotechnol 2010;87(1):309-17.

12. Römling $U$, Balsalobre C. Biofilm infections, their resilience to therapy and innovative treatment strategies. J Intern Med 2012;272(6):541-61.

13. Kim HS. Do not put too much value on conventional medicines. J Ethnopharmacol 2005;100(1-2):37-9.

14. Palombo EA. Phytochemicals from traditional medicinal plants used in the treatment of diarrhoea: Modes of action and effects on intestinal function. Phytother Res 2006;20:717-24.

15. Namsivayam KR, Roy EA. Anti-biofilm effect of medicinal plant extracts against clinical isolate of biofilm of Escherichia coli. Int $\mathrm{J}$ Pharm Pharm Sci 2013;5(2):486-9.
16. Kusum H, Anju B, Ravi KG, Raghika S. Leaf extract of Azadirachta indica (neem): A potential antibiofilm agent for Pseudomonas aeruginosa. Pathog Dis 2013;69:62-5.

17. Mundada S, Shivhare R. Pharmacology of Tridax procumbens a weed: Review. Int J PharmTech Res 2010;2(2):1391-4.

18. Darsini DT, Srinivasan P, Guna G, Manimekalai K, Dineshbabu J. In vitro antibiofilm activity of Piper longum and Piper nigrum against clinical isolates of Sterptococcus pyogens isolated from Pharyngitis patients. Int Res J Pharm 2015;6(2):122-32.

19. Abdullah Al-Dhabi N, Valan Arasu M, Rejiniemon TS. In vitro antibacterial, antifungal, antibiofilm, antioxidant, and anticancer properties of isosteviol isolated from endangered medicinal plant Pittosporum tetraspermum. Evid Based Complement Alternat Med 2015;2015:164261.

20. Al-Dhabi NA, Balachandran C, Raj MK, Duraipandiyan V, Muthukumar C, Ignacimuthu S, et al. Antimicrobial, antimycobacterial and antibiofilm properties of Couroupita guianensis Aubl. Fruit extract. BMC Complement Altern Med 2012;12:242.

21. Kazemian H, Ghafourian S, Heidari H, Amiri P, Yamchi JK, Shavalipour A, et al. Antibacterial, anti-swarming and anti-biofilm formation activities of Chamaemelum nobile against Pseudomonas aeruginosa. Rev Soc Bras Med Trop 2015;48:432-6.

22. Grujic SM, Radojevic ID, Vasic SM, Comic LJ, Topuzovic M. Antimicrobial and antibio film activities of secondary metabolites from Vinca minor L. Appl Biochem Microbiol 2015;51(5):572-8.

23. Budzynska A, Wieckowska-Szakiel M, Sadowska B, Kalemba D, Rózalska B. Antibiofilm activity of selected plant essential oils and their major components. Pol J Microbiol 2011;60(1):35-41.

24. Jalal Z, El Atki Y, Lyoussi B, Abdellaoui A. Photochemistry of the essential oil of Melissa officinalis L. growing wild in Morocco: Preventive approach against nosocomial infections. Asian Pac J Trop Biomed 2015;5(6):458-61.

25. Awolola GV, Koorbanally NA, Chenia H, Shode FO, Baijnath H. Antibacterial and anti-biofilm activity of flavonoids and triterpenes isolated from the extracts of Ficus sansibarica Warb. subsp. sansibarica (Moraceae) extracts. Afr J Tradit Complement Altern Med 2014;11(3):124-31

26. Sangam S, Rao DV, Sharma RA. A review on Pongamia pinnata (L.) pierre: A great versatile leguminous plant. Nat Sci 2010;8(11):1309.

27. Ali BH, Blunden G, Tanira MO, Nemmar A. Some phytochemical, pharmacological and toxicological properties of ginger (Zingiber officinale Roscoe): A review of recent research. Food Chem Toxicol 
2008;46(2):409-20.

28. Bezalwar PM, Shuddhalwar PP. A quest of anti-biofilm activity of Zingiber officinale root and Coriandrum sativum seed extract against clinical isolates of Pseudomonas aeruginosa and Staphylococcus auerus. Int J Sci Res 2013;4(4):2581-4

29. Dineshbabu J, Srinivasan P, Darsini TP, Srinivasan P, Everlyne IM, Manimekalai K. Synergistic anti-biofilm activity of medicinal plants against biofilm forming Streptococcus pyogenes from pharyngitis patients. Indo Am J Pharm Res 2015;5(8):2598-606.

30. Sukunlaya S, Jindaporn P, Rawee T. Anticandida and antibiofilm activity of Artocarpus lakoocha extract. Songklanakarin J Sci Technol 2014:36(4):451-7.

31. Hossain MF, Islam MA, Akhtar S, Numan SM. Nutritional value and medicinal uses of monkey jack fruit (Artocarpus lakoocha). Int Res J Biol Sci 2016;5(1):60-3.

32. Handral HK, Pandith A, Shruthi SD. A review on Murraya koenigii: Multipotential medicinal plant. Asian J Pharm Clin Res 2012;5(4):5-14

33. Ganesh PS, Vital RR. In vitro antibiofilm activity of Murraya koenigii essential oil extracted using supercritical fluid $\mathrm{CO} 2$ method against Pseudomonas aeruginosa PAO1. Nat Prod Res 2015;29(24):2295-8.

34. Luís A, Breitenfeld L, Ferreira S, Duarte AP, Domingues F. Antimicrobial, antibiofilm and cytotoxic activities of Hakea sericea Schrader extracts. Pharmacogn Mag 2014;10 Suppl 1:S6-13.

35. Yahya MF, Ibrahim MS, Zawawi WM, Hamid UM. Biofilm killing effects of Chromolaena odokrata extracts against Pseudomonas aeruginosa. Res J Phytochem 2014;8(3):64-73.

36. De Araujo AR, Quelemas PV, Perfeito ML, Lima LI, Sa MC, Nunes PH, et al. Antibacterial, antibiofilm and cytotoxic activities of Terminalia fagifolia Mart. extract and fractions. Ann Clin Microbiol Antimicrob 2015;14:25.

37. De Queiroz AC, de Lira DP, Dias Tde L, de Souza ET, da Matta CB, de Aquino $\mathrm{AB}$, et al. The antinociceptive and anti-inflammatory activities of Piptadenia stipulacea Benth. (Fabaceae). J Ethnopharmacol 2010;128:377-83.

38. Silva JF, Silva LC, Arruda IR, Arruda IR, Silva AG, Macedo AJ, et al. Antimicrobial activity of Pityrocarpa monilifomis leaves and its capacity to enhance the activity of four antibiotics against Staphylococcus aureus strains. J Med Plants Res 2013;7:2067-72.

39. Trentin DS, Zimmer KR, Silva MV, Raquel BG, Alexandre JM. Medicinal plants from Brazilian Caatinga: Antibiofilm and antibacterial activities against Pseudomonas aeruginosa. Rev Caatinga 2014;27(3):264-71.

40. Muruzovic MZ, Mladenovic KG, Stefanovic OD, Vasic SM, Comic LR. Extracts of Agrimonia eupatoria L. as sources of biologically active compounds and evalution of their antioxidant, antimicrobial and antibiofilm activities. J Food Drug Anal 2016;24:539-47.

41. Sosa V, Zunino P. Effect of Ibicella lutea on uropathogenic Proteus mirabilis growth, virulence, and biofilm formation. J Infect Dev Ctries 2009:3:762-70.

42. Goncalves AL, Filho AA, Menezes H. Antimicrobial effects of some Brazilian medicinal plants on intestinal disorders. Artig Orig 2011;4(2):153-60

43. Abinaya M, Gayathri M, Khanna VG. Evalution of in-vitro biological and antibiofilm activities of various leaf extracts of Aerva lanata. Int $\mathrm{J}$ Pharm Sci Rev Res 2016;38(1):65-7.

44. Anita A, Retna AM. Review on the medicinal plants Aerva lanata. Asian J Biochem Pharm Res 2013;1(3):2231-560.

45. Carbonari KA, Ferreira EA, Rebello JM, Felipe KB, Rossi MH, Felício JD, et al. Free-radical scavenging by Ouratea parviflora in experimentally-induced liver injuries. Redox Rep 2006;11:124-30.

46. Trentin Dda S, Giordani RB, Zimmer KR, da Silva AG, da Silva MV, Correia MT, et al. Potential of medicinal plants from the Brazilian semiarid region (Caatinga) against Staphylococcus epidermidis planktonic and biofilm lifestyles. J Ethnopharmacol 2011;137:327-35.

47. Mary R, Banu N. Screening of anti-biofilm, anti-quorum sensing activity from methanolic extract of Plectranthus amboinicus (Lour). Int J Pharm Bio Sci 2015;6(4):1060-8.

48. D'Abrosca B, Buommino E, D'Angelo G, Coretti L, Scognamiglio M, Severino V, et al. Spectroscopic identification and anti-biofilm properties of polar metabolites from the medicinal plant Helichrysum italicum against Pseudomonas aeruginosa. Bioorg Med Chem 2013;21:7038-46.

49. Antunes Viegas D, Palmeira-de-Oliveira A, Salgueiro L, Martinez-de-Oliveira J, Palmeira-de-Oliveira R. Helichrysum italicum: From traditional use to scientific data. J Ethnopharmacol
2014;151(1):54-65.

50. Wojnicz D, Kucharska AZ, Letowska AS, Sokół-Łętowska A, Kicia M, Tichaczek-Goska D. Medicinal plants extracts affect virulence factors expression and biofilm formation by the uropathogenic Escherichia coli. Urol Res 2012;40:683-97.

51. Perumal S, Mahmud R. Chemical analysis, inhibition of biofilm formation and biofilm eradication potential of Euphorbia hirta L. against clinical isolates and standard strains. BMC Complement Altern Med 2013;13:346.

52. Tang Y, Lou Z, Rahman RT, Al-Hajj NQ, Wang H. Chemical composition and anti-biofilm activity of burdock (Arctium lappa $\mathrm{L}$ Asteraceae) leaf fractions against Staphylococcus aureus. Trop J Pharm Res 2014;13(11):1933-9.

53. Chan YS, Cheng LN, Wu JH, Chan E, Kwan YW, Lee SM, et al. A review of the pharmacological effect of Arctium lappa (Burdock). Inflammopharmacology 2011;19(5):245-54.

54. Kamonwannasit S, Nantapong N, Kumkrai P, Luecha P, Kupittayanant S, Chudapongse N. Antibacterial activity of Aquilaria crassna leaf extract against Staphylococcus epidermidis by disruption of cell wall. Ann Clin Microbiol Antimicrob 2013 20;12:20.

55. Avoseh O, Oyedeji O, Rungqu P, Nkeh-Chungag B, Oyedeji A. Cymbopogon species; ethnopharmacology, phytochemistry and the pharmacological importance. Molecules 2015;20:7438-53.

56. Mohsenipour Z, Hassanshahian M. The effects of Allium sativum extracts on biofilm formation and activities of six pathogenic bacteria. Jundishapur J Microbiol 2015;8:e18971.

57. Al-Bakri AG, Othman G, Afifi FU. Determination of the antibiofilm, antiadhesive, and anti-MRSA activities of seven Salvia species. Pharmacogn Mag 2010;6(24):264-70.

58. Murugan K, Selvanayaki K, Al-Sohaibani S. Antibiofilm activity of Andrographis paniculata against cystic fibrosis clinical isolate Pseudomonas aeruginosa. World J Microbiol Biotechnol 2011;27:1661-8.

59. Abbas H, Bayomi IM, Moustafa MZ. In vitro antibacterial and antibiofilm activities of Hibiscus sabdariffa L. extracts and apple vinegar against bacteria isolated from diabetic foot infections. Res J Pharm Technol 2014;7(2):131-6.

60. Puro K, Sunjukta R, Samir S, Ghatak S, Shakuntala I, Sen A. Medicinal uses of Rosella plant (Hibiscus sabdariffa L): Mini review. Indian J Hill Farming 2014;27(1):81-90

61. Karicheri R, Antony B. Antibacterial and antibiofilm activities of Peppermint (Mentha piperita Linn) and methanol mint (Mentha arvensis Linn) essential oils on Aggregatibacter actinomycetemcomitans isolated from orodental infections. Eur J Pharm Med Res 2016;3(7):577-81.

62. Saharkhiz MJ, Motamedi M, Zomorodian K, Pakshir K, Miri R, Hemyari K. Chemical composition, antifungal and antibiofilm activities of the essential oil of Mentha piperita L. ISRN Pharm 2012;2012:718645.

63. Mutalib LY, Nuraddin SM, Aka TH. Phytochemical screening, antibacterial and antibiofilm evaluation of Lagenaria siceraria fruit growing in Kurdistan region/Iraq. J Pharmacogn Phytochem 2015;4(1):45-9.

64. Pattnaik A, Sarkar R, Sharma A, Yadav KK, Kumar A, Roy P, et al. Pharmacological studies on Buchanania lanzan Spreng.- A focus on wound healing with particular reference to anti-biofilm properties. Asian Pac J Trop Biomed 2013;3:967-74.

65. Ghaima KK, Rasheed AF, Ahmed EF. Antibiofilm, antibacterial and antioxidant activities of water extracts of Calendula officinalis flowers. Int J Biol Pharm Res 2013;4(7):465-70.

66. Muley BP, Khadabadi SS, Banarase NB. Phytochemical constituents and pharmacological activities of Calendula officinalis (Asteraceae): A review. Trop J Pharm Res 2009;8(5):455-65.

67. Zivkovic J, Stojkovic D, Petrovic J, Zdunić G, Glamočlija J, Soković M. Rosa canina L.-New possibilities for an old medicinal herb. Food Funct 2015;6(12):3687-92.

68. Limsuwan S, Voravuthikunchai SP. Boesenbergia pandurata (Roxb) Schltr. Eleutherine Americana Merr. and Rhodomyrtus tomentosa (Aiton) Hasskas antibiofilm producing and antiquorum sensing in Streptococcus pyogenes. FEMS Immunol Med Microbiol 2008;53:429-436.

69. Rozalski M, Micota B, Sadowska B, Stochmal A, Jedrejek D, Wieckowska-Szakiel M, et al. Antiadherent and antibiofilm activity of Humulus Lupulus L. derived products: New pharmacological properties. BioMed Res Int 2013;2013:7.

70. Koettar U, Biendi M. Hops (Humulus lupulus): A review of its historic 
and medicinal uses. Am Bot Council 2010;87:44-57.

71. Rejiniemon TS, Arasu MV, Duraipandiyan V, Ponmurugan K, AlDhabi NA, Arokiyaraj S. In-vitro antimicrobial, antibiofilm, cytotoxic, antifeedant and larvicidal properties of novel quinone isolated from Aegle marmelos (Linn.) Correa. Ann Clin Microbiol Antimicrob
2014;13:48

72. Pratiwi SU, Lagendijk EL, Hertiani T, De Weert S, Van Den Hondel CA. Antimicrobial effects of Indonesian medicinal plants extracts on planktonic and biofilm growth of Pseudomonas aeruginosa and Staphylococcus aureus. Int J Pharm Pharm Sci 2015;7(4):183-91. 